\title{
New Macroscopic Ferrimagnets in the System Co-TbN
}

\author{
Taewan Kim ${ }^{1 *}$ and Jung Keun $\mathrm{Oh}^{2}$ \\ ${ }^{1}$ Department of Advanced Materials Engineering, Sejong University, Seoul 143-747, Korea \\ ${ }^{2}$ College of Architecture, Konkuk University, Seoul 143-701, Korea
}

(Received 4 March 2008)

\begin{abstract}
This study examines a new macroscopic ferrimagnet, Co-TbN. This ferrimagnet, consisting of two metallic phases, Co and $\mathrm{TbN}$, demonstrated the typical macroscopic ferrimagnet properties of a magnetic compensation point and a negative giant magnetoresistance (GMR). The Co-TbN system with $32 \%$ TbN composition showed $0.72 \%$ GMR in magnetic fields up to $8 \mathrm{kOe}$ at room temperature and $9 \%$ GMR in $40 \mathrm{kOe}$ at $250 \mathrm{~K}$. In the Co-TbN system, GMR exhibited a different dependence on temperature from that of ordinary GMR materials whose negative magnetoresistance decreases with increasing temperature. In contrast to ordinary GMR materials whose negative magnetoresistance decreases with increasing temperature, the GMR effect in the CoTbN system increased with increasing temperature, due to the increase of ferromagnetic alignment of the Co and $\mathrm{TbN}$ in the magnetic field caused by the decreased exchange coupling with increasing temperature.
\end{abstract}

Keywords : macroscopic ferrimagnet, antiparallel exchange, magnetic compensation giant magnetoresistance

\section{Introduction}

Macroscopic ferrimagnets are a newly discovered class of phase-separated magnetic materials [1]. They consist of two magnetic phases with a negative magnetic exchange at the phase boundary. A prototypical example is the CoEuS system which has $100 \AA$ particles of EuS in a Co matrix. The EuS is exchange coupled antiferromagnetically to the Co at least at the $\mathrm{Co} / \mathrm{EuS}$ interface. In the CoEuS system, the small size of the EuS particles provides a large fraction of the EuS in close proximity to the interface which is influenced by the strong $\mathrm{Co} / \mathrm{EuS}$ exchange. These materials have been found to display unusual magneto-optical [2] and magneto-transport [3,4] properties. Magnetization and Kerr hysteresis loops have confirmed the macroscopic ferrimagnetic model for these systems. In measurements of the optical and magneto-optical properties of Co-EuS thin films, polar Kerr rotations of up to $2^{\circ}$ have been observed in Co rich films at photon energies of $4.5 \mathrm{eV}$ [5]. Transport measurements show that the magnetoresistance of Co-EuS behaves like that of the widely studied, granular giant magnetoresistance (GMR) materials [6] which consist of particles of a ferromagnetic metal in a conductive, nonmagnetic matrix. In contrast,

*Corresponding author: Tel: +82-2-3408-3691

Fax: +82-2-3408-3664, e-mail: twkim@ @ejong.ac.kr
Co-EuS consists of semiconducting, ferromagnetic particles in a conductive, ferromagnetic matrix of Co. As a consequence, the temperature dependence of the magnetoresistance is very different in the Co-EuS system as compared to that of the ordinary granular GMR materials. With respect to the magnitude of the effect, the magnetoresistivity change $(\mathrm{dr})$ of the Co-EuS system is $8 \times 10^{-5}$ $\Omega \mathrm{cm}$, which is larger than that of other magnetoresistive materials. Even though the magnetoresistivity change of this system is large, the magnetoresistance defined as $\delta \rho /$ $\rho$ is small, typically $2 \sim 3 \%$, because of the high resistivity of the material caused by a large volume fraction of semiconducting EuS phase.

This study proposed a new macroscopic ferrimagnet, Co-TbN, with $\mathrm{TbN}$ precipitates in a Co matrix (Fig. 1). $\mathrm{TbN}$ has the same atomic magnetic moment as pure $\mathrm{Tb}$ and the same rock salt structure as EuS [7]. The TbN precipitates also provide a higher Curie temperature and thus stronger antiparallel exchange coupling with the Co matrix than EuS does. These stronger exchange effects are caused by the RKKY-type exchange mediated by conduction electrons [8], which is weak in semiconducting EuS. Another difference is the single ion anisotropy of the $\mathrm{Tb}$ atom which is a non-S-state ion. In contrast, EuS contains divalent europium which is an S-state ion and thus has zero, single-ion anisotropy. Furthermore, $\mathrm{TbN}$ is a conductor rather than a semiconductor so the resistivity 


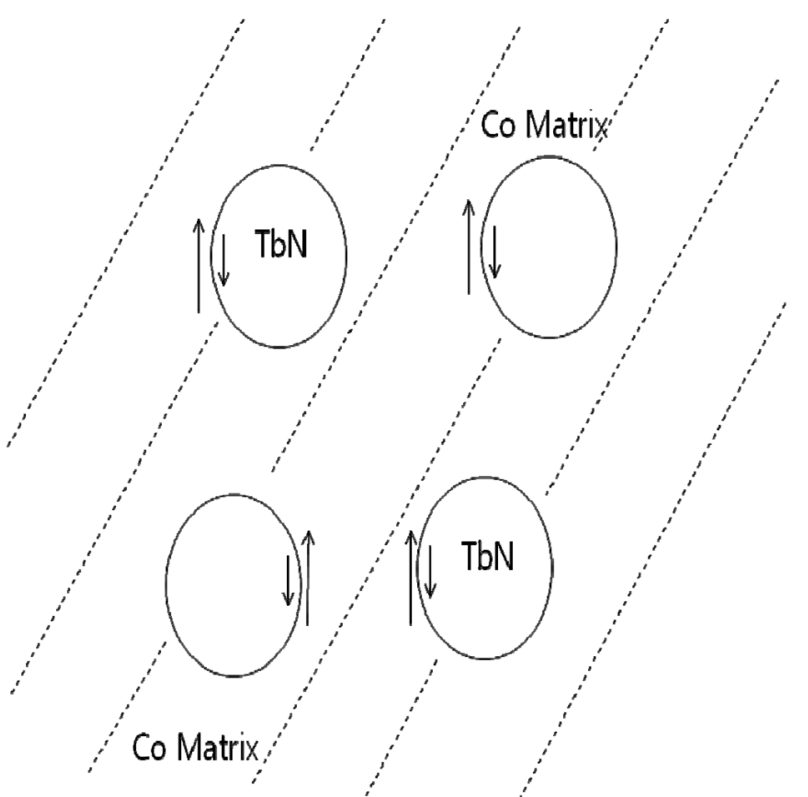

Fig. 1. Simple schematic diagram illustrating antiparallel exchange coupling at the phase boundary in the typical CoTbN system.

of $\mathrm{Co}-\mathrm{TbN}$ is much less than that of $\mathrm{Co}-\mathrm{EuS}$, which can improve the magnetoresistance, $\delta \rho / \rho$. Co-TbN differs from the granular GMR materials in that both phases are magnetic and also differs from Co-EuS in that both phases are conductors.

\section{Experiments}

Amorphous $\mathrm{Tb}_{\mathrm{x}} \mathrm{Co}_{1-\mathrm{x}}$ thin films were prepared with $\mathrm{x}=$ $25 \sim 32 \%$ by magnetron sputtering system with a composite target. The film compositions were controlled by controlling the Ar sputtering gas pressure in the range 5 15 mTorr. Nitrogen was introduced into the films by annealing at 400 and $650^{\circ} \mathrm{C}$ for 12 hours with a continuous flow of $10 \% \mathrm{H}_{2}$-balance $\mathrm{N}_{2}$ gas mixture in order to induce the phase separation of $\mathrm{Co}$ and $\mathrm{TbN}$. Phase analysis was conducted with X-ray diffraction (XRD; $1.542 \AA \mathrm{Cu} \mathrm{K} \mathrm{K}_{\alpha}$ radiation and tube operating at $40 \mathrm{kV}$ and $30 \mathrm{~mA}$ ) and secondary electron images on a field emission scanning electron microscope (FE-SEM). Magnetization loops and magnetization vs. temperature plots were made in the temperature range $-100^{\circ} \mathrm{C}$ to $400^{\circ} \mathrm{C}$ and in magnetic fields up to $13 \mathrm{kOe}$ using a vibrating sample magnetometer. The magnetization loops and magnetization vs. temperature plots in high magnetic fields up to $30 \mathrm{kOe}$ were measured at temperatures from $20 \mathrm{~K}$ to $300 \mathrm{~K}$ using a SQUID magnetometer. Magnetoresistance and Hall effect measurements at room temperature were made in fields up to $8.5 \mathrm{kOe}$ with a DC electromagnet using Van der Pauw geometry. Contacts were made with fine wires attached with silver paint at the corners of a square sample. The magnetoresistance with temperature experiment was performed in fields up to $40 \mathrm{kOe}$ over the temperature range from $20 \mathrm{~K}$ to $250 \mathrm{~K}$ in a superconducting coil cryostat.

\section{Results and Discussion}

3.1. Changes of Magnetic Properties and Hall Effect by the Phase Separation of Co-TbN in Amorphous TbCo Thin Films

Amorphous TbCo thin films were transformed to $\mathrm{Co}$ and $\mathrm{TbN}$ by annealing in a $\mathrm{N}_{2}$ gas atmosphere where the phase transformation was induced by the diffusion of nitrogen atoms at different temperatures. The diffraction pattern from the sample annealed at $400^{\circ} \mathrm{C}$ (Fig. 2) exhibited the formation of two phases: Co and $\mathrm{TbN}$. The Co phase had sharp primary peaks but the $\mathrm{TbN}$ phase had broad primary peaks. The amorphous $\mathrm{Tb}_{0.32} \mathrm{Co}_{0.68}$ thin films before annealing had high perpendicular easy axis anisotropy (Fig. 3) and showed a large negative Hall effect, $\rho_{\mathrm{H}}=-2.14 \times 10^{-6} \Omega \mathrm{cm}$ (Fig. 4). On the other hand, the sample phase annealed at $400^{\circ} \mathrm{C}$ and separated into $\mathrm{Co}$ and $\mathrm{TbN}$ displayed a bubble domain-type, magnetic loop with much lower perpendicular easy axis anisotropy (Fig. 5) and a positive Hall resistivity (Fig. 6). These changes resulted from the phase transformation from amorphous $\mathrm{TbCo}$ to a polycrystalline mixture of $\mathrm{Co}$ and $\mathrm{TbN}$. As the Tb content in the Co phase decreased due to

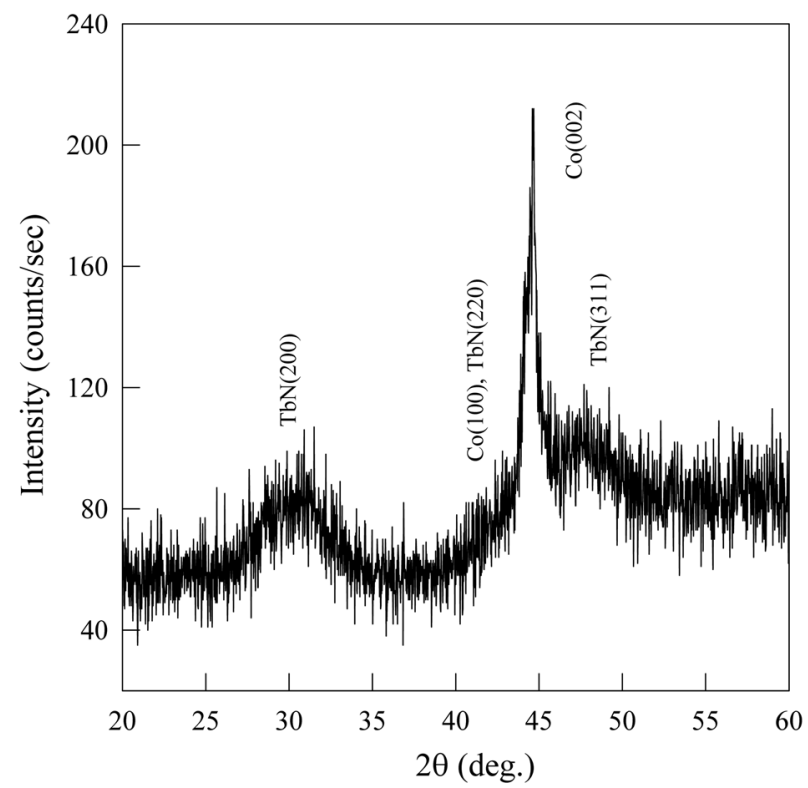

Fig. 2. The $\mathrm{X}$-ray diffraction pattern of the TbCo films annealed at $400^{\circ} \mathrm{C}$ in a $\mathrm{N}_{2}$ atmosphere. 

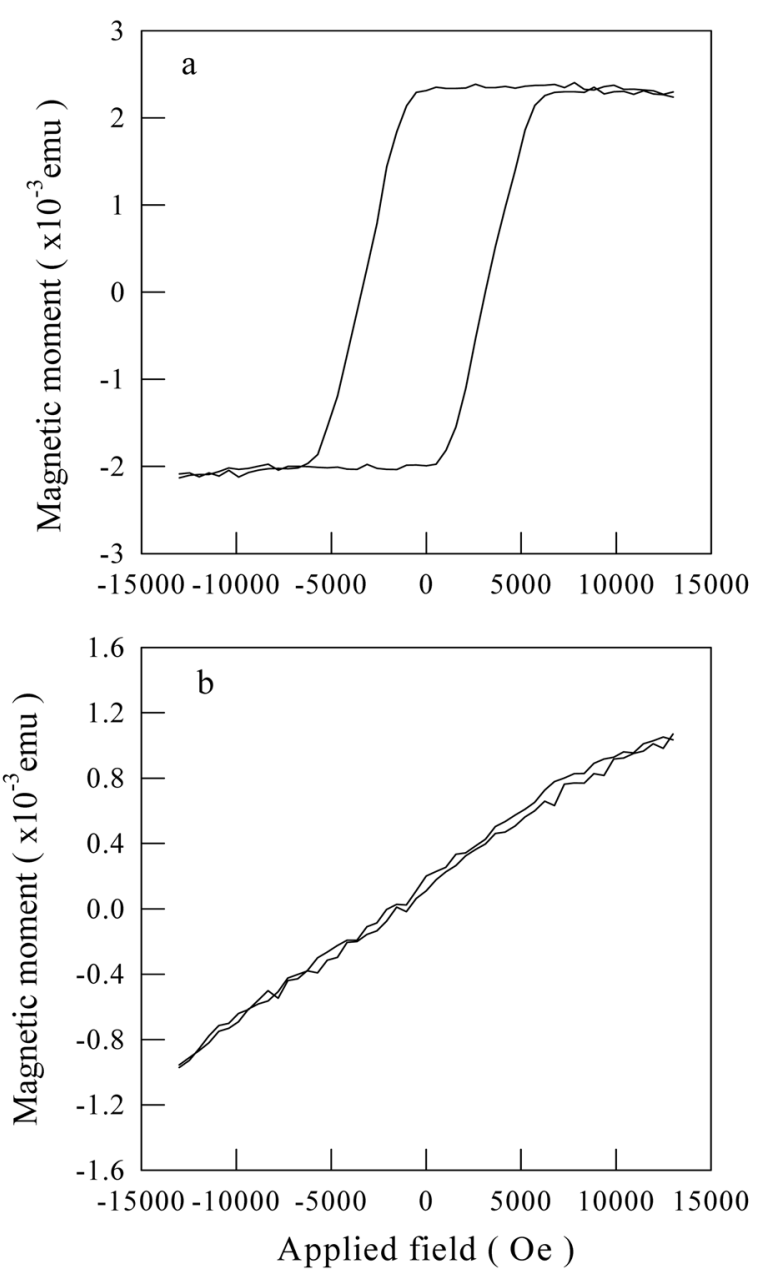

Fig. 3. The out-of-plane (a) and in-plane (b) magnetic moment changes of the as-deposited, amorphous, $\mathrm{Tb}_{0.32} \mathrm{Co}_{0.68}$ thin film.

the formation of $\mathrm{TbN}$, the perpendicular anisotropy decreased and the Hall sign changed from negative to positive. The Hall resistivity of the sample annealed at $400^{\circ} \mathrm{C}$ retained a high value of $3 \times 10^{-7} \Omega \mathrm{cm}$ compared with the small Hall resistivity of $1.07 \times 10^{-8} \Omega \mathrm{cm}$ for the pure polycrystalline $\mathrm{Co}$. This indicated that the $\mathrm{Tb}$ was not completely separated from the Co and that some was still dissolved in the Co phase.

When the films were annealed at the higher temperature of $650{ }^{\circ} \mathrm{C}$, the microstructure and magnetic properties were completely different from those of the film annealed at the lower temperature $\left(400^{\circ} \mathrm{C}\right)$. As the sample was annealed at the higher temperature, $\mathrm{TbN}$ became the primary phase and precipitated out of the Co matrix. The in-plane SEM pictures indicated that the $\mathrm{TbN}$ phase formed large $(>20 \mu \mathrm{m})$ and small $(<0.5 \mu \mathrm{m})$ precipitates in the Co matrix. The small precipitates were probably segregated in the grain boundaries of the Co matrix. Fig. 7 shows the diffraction pattern of the film annealed at the

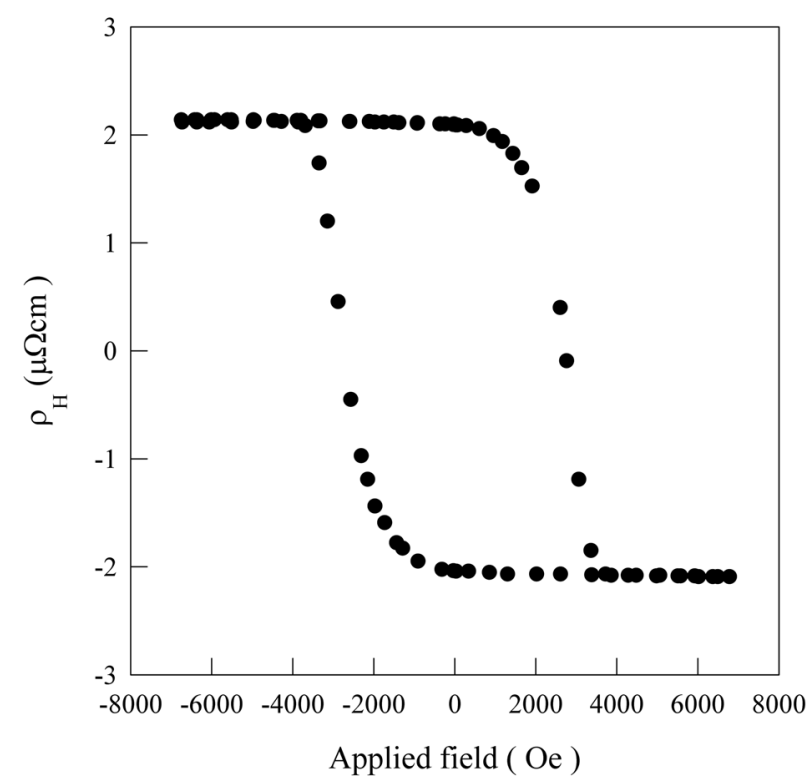

Fig. 4. The negative Hall effect of the as-deposited, amorphous, $\mathrm{Tb}_{0.32} \mathrm{Co}_{0.68}$ thin film.
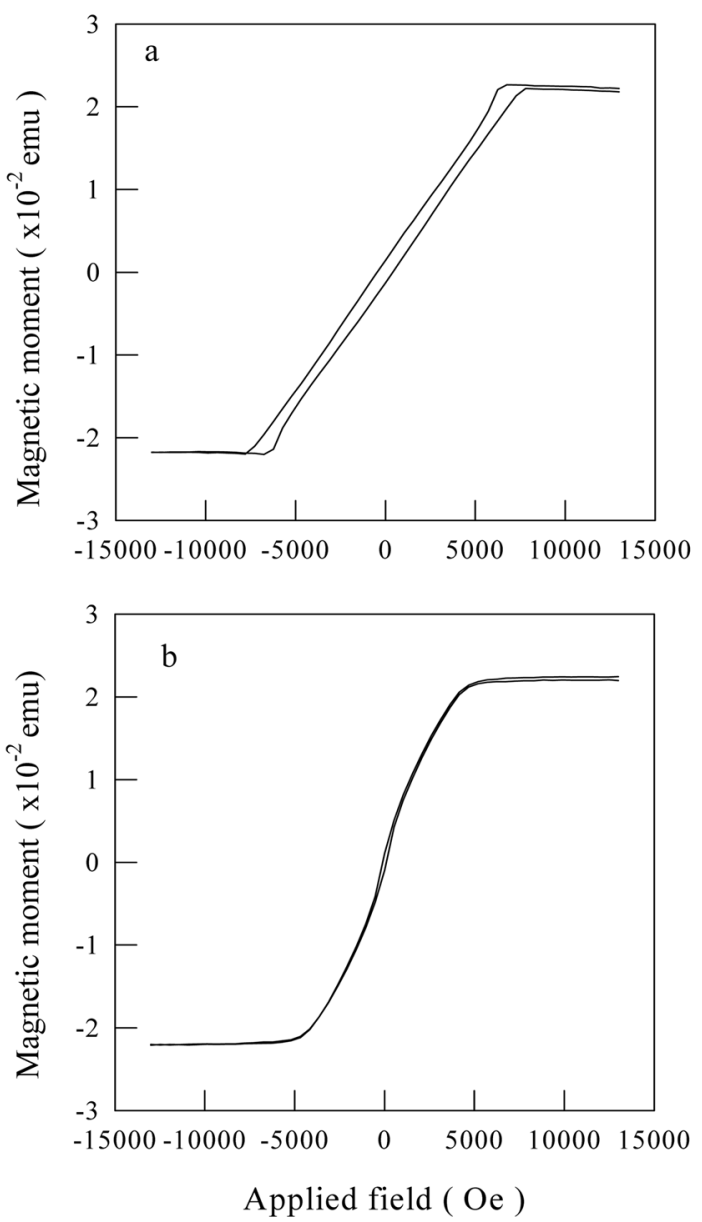

Fig. 5. The out-of-plane (a) and in-plane (b) magnetic moment changes of the $\mathrm{Tb}_{0.32} \mathrm{Co}_{0.68}$ film annealed at $400^{\circ} \mathrm{C}$ in a $\mathrm{N}_{2}$ atmosphere. 


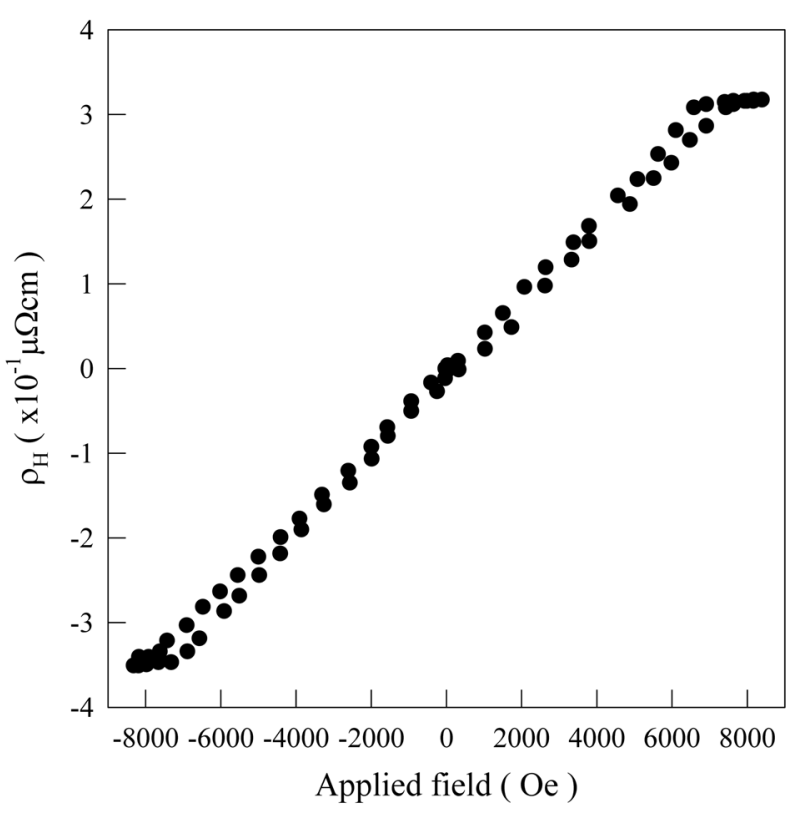

Fig. 6. The positive Hall effect of the $\mathrm{Tb}_{0.32} \mathrm{Co}_{0.68}$ film annealed at $400^{\circ} \mathrm{C}$ in a $\mathrm{N}_{2}$ atmosphere.

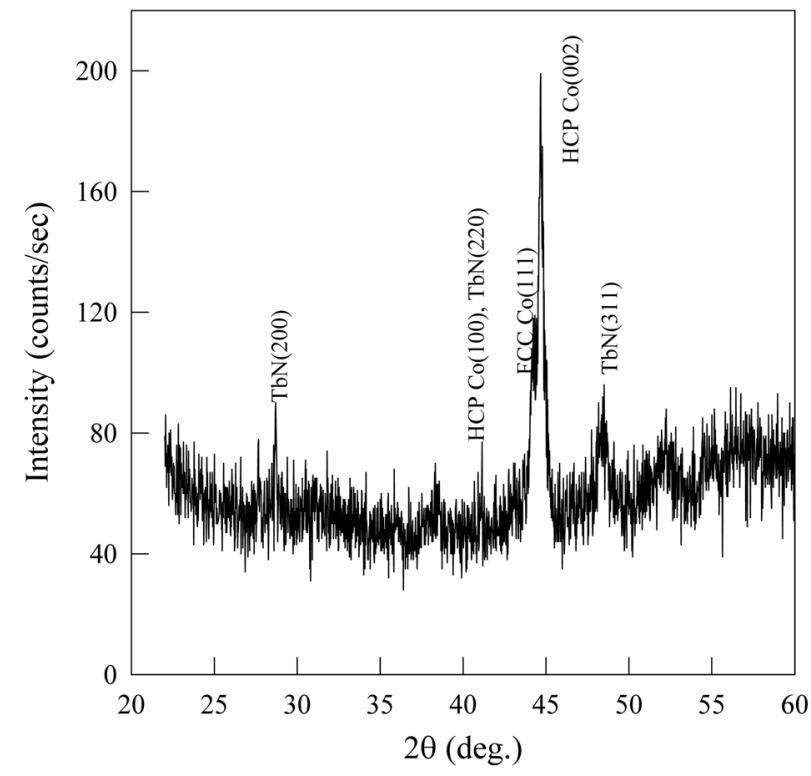

Fig. 7. The $\mathrm{X}$-ray diffraction pattern of the TbCo film annealed at $650^{\circ} \mathrm{C}$ in a $\mathrm{N}_{2}$ atmosphere.

higher temperature which had stronger $\mathrm{TbN}$ peaks. The diffraction pattern also exhibited the primary peaks of face-centered cubic (FCC) and hexagonal close-packed (HCP) Co structures which indicated that the Co matrix consisted of two structures: FCC and HCP. The sample annealed at the higher temperature showed in-plane easy axis anisotropy rather than perpendicular easy axis anisotropy (Fig. 8) and the Hall resistivity almost disappeared. The changes in microstructure and magnetic property,
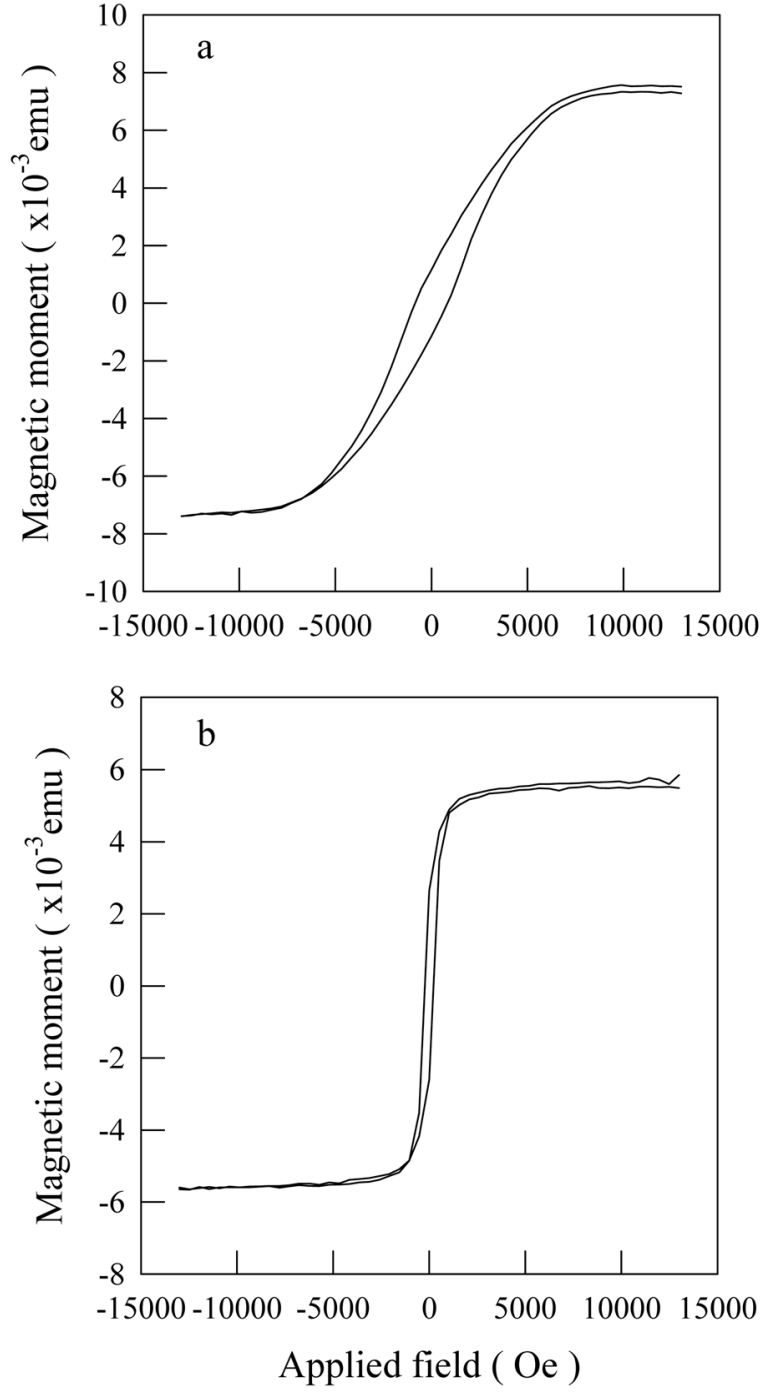

Fig. 8. The out-of-plane (a) and in-plane (b) magnetic moment changes of the $\mathrm{Tb}_{0.32} \mathrm{Co}_{0.68}$ film annealed at $650^{\circ} \mathrm{C}$ in a $\mathrm{N}_{2}$ atmosphere.

along with the insignificant Hall effect, of the sample annealed at $650^{\circ} \mathrm{C}$ indicate that the amorphous $\mathrm{TbCo}$ thin films had been almost fully transformed to $\mathrm{TbN}$ precipitates and a Co matrix.

Figs. 9a and $\mathrm{b}$ show the saturation magnetic moment and coercivity changes as a function of temperature in the Co-TbN film with the composition of $32 \% \mathrm{TbN}$, which is typical for the Co-TbN macroscopic ferrimagnets. The saturation magnetic moment curve showed a small break at about $150^{\circ} \mathrm{C}$ in the center, which suggested the presence of two different magnetic phases with different Curie temperatures in the macroscopic ferrimagnet. The coercivity increased with increasing temperature between $0^{\circ} \mathrm{C}$ and $200^{\circ} \mathrm{C}$, which was very unusual behavior. The dividing point between the low and high coercivity regions 

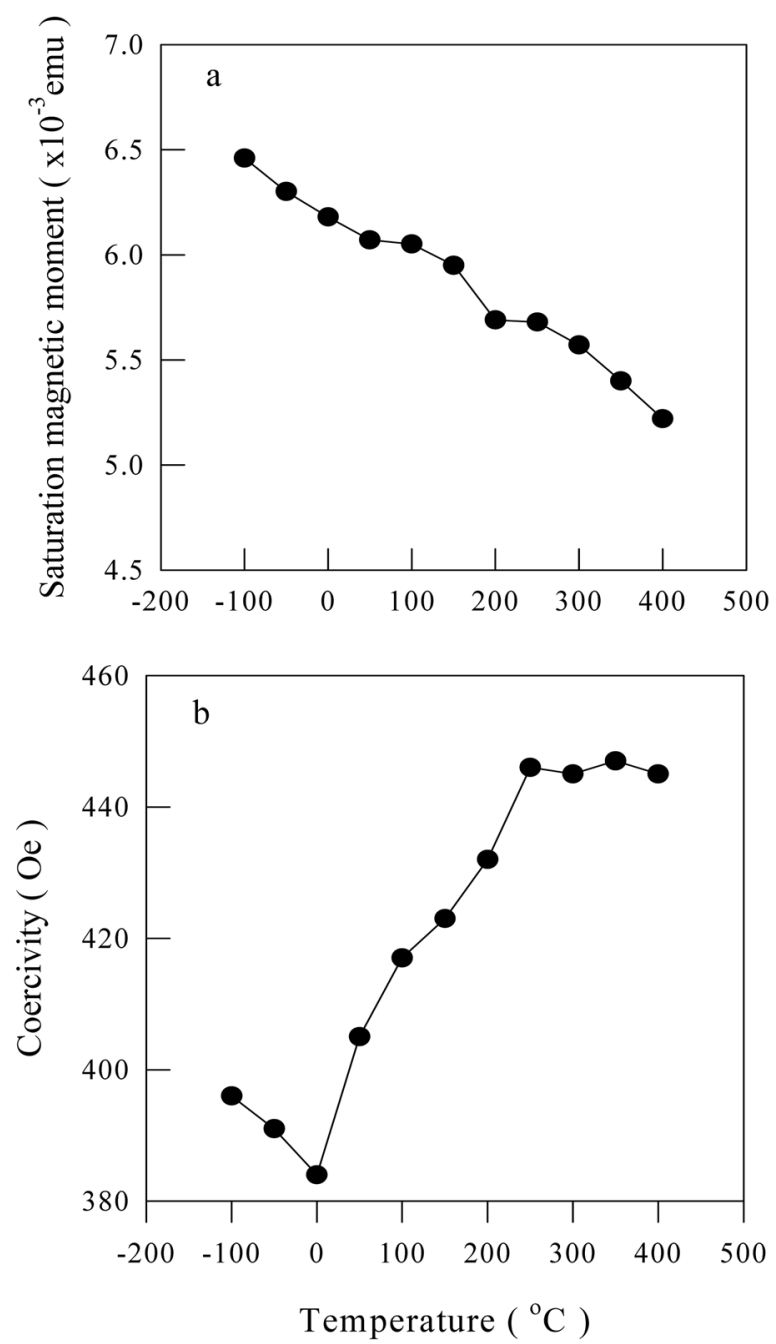

Fig. 9. The saturation magnetic moment (a) and coercivity (b) changes as a function of temperature in the $\mathrm{Tb}_{0.32} \mathrm{Co}_{0.68}$ film annealed at $650^{\circ} \mathrm{C}$ in a $\mathrm{N}_{2}$ atmosphere.

was about $150^{\circ} \mathrm{C}$, suggesting a connection with the break in the magnetization vs. temperature curve. We suggest that the magnetization processes at low and high temperatures take place via different mechanisms because both $\mathrm{Co}$ and $\mathrm{TbN}$ are magnetically ordered at low temperatures whereas only $\mathrm{Co}$ is ferromagnetic at high temperatures.

\subsection{Giant Magnetoresistance (GMR) Effect of Co- TbN Macroscopic Ferrimagnets}

The magnetic and magneto-transport properties obtained from the Co-TbN film with $32 \% \mathrm{TbN}$ composition are shown in Figs. 10 to 13. These data were typical of the magnetic behavior for all compositions from $25 \%$ to $32 \%$ $\mathrm{TbN}$. The increase in magnetic moment with increasing temperature and the broad minimum in magnetic moment in a field of $1 \mathrm{kOe}$ were clear indications of ferrimagnetic behavior. The small jump in the magnetic moment curves

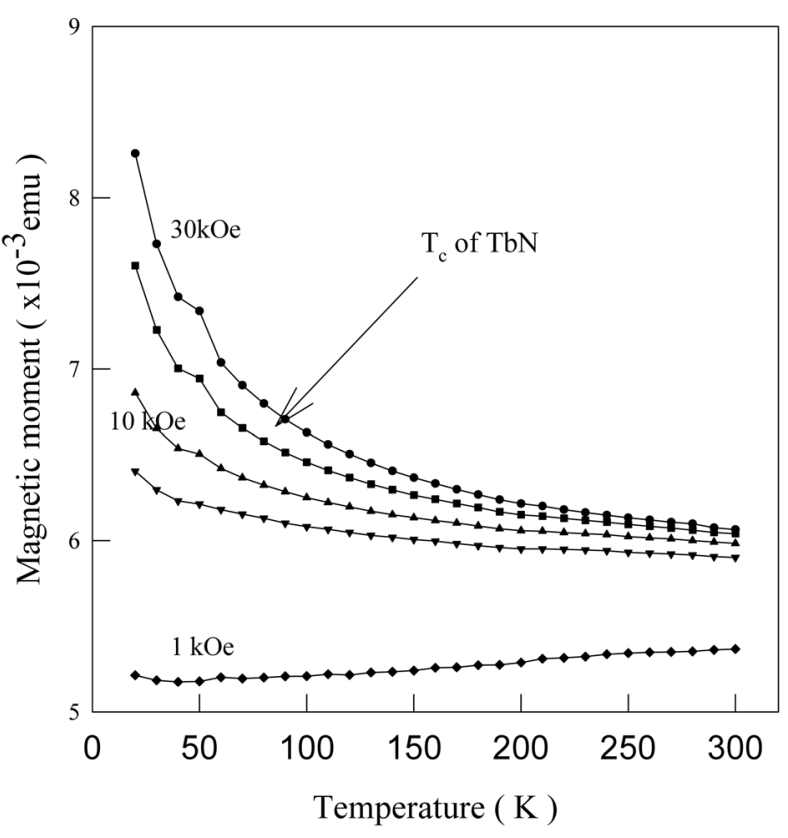

Fig. 10. The magnetic moment vs. temperature of $\mathrm{Co}_{0.68^{-}}$ $(\mathrm{TbN})_{0.32}$ at various applied fields.

in fields of 10,20 and $30 \mathrm{kOe}$ at $50 \mathrm{~K}$ indicated that at these high field strengths the magnetic moments of $\mathrm{TbN}$ precipitates were ferromagnetically aligned with the Co matrix. The Curie temperature of $\mathrm{TbN}$ was estimated to be about $75 \mathrm{~K}$ by extrapolating from the break in the magnetization curve in the $30 \mathrm{kOe}$ field. Fig. 11 shows the magnetic moment curves of the $\mathrm{Co}_{0.68}-(\mathrm{TbN})_{0.32}$ film with applied field at different temperatures. The magneti-

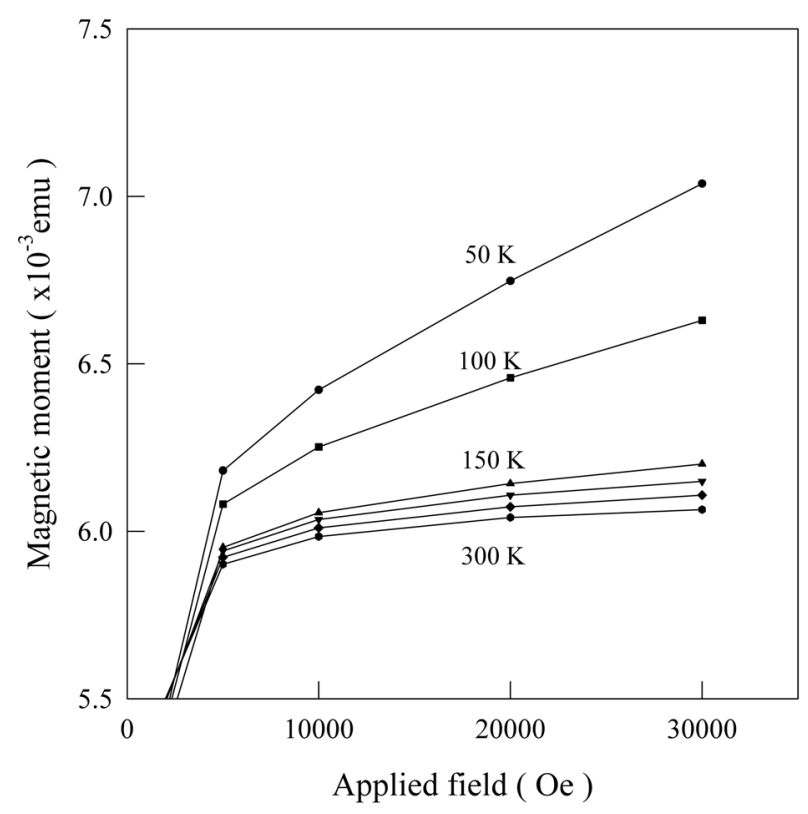

Fig. 11. The magnetic moment vs. applied field of the Co-TbN macroscopic ferrimagnet at various temperatures. 

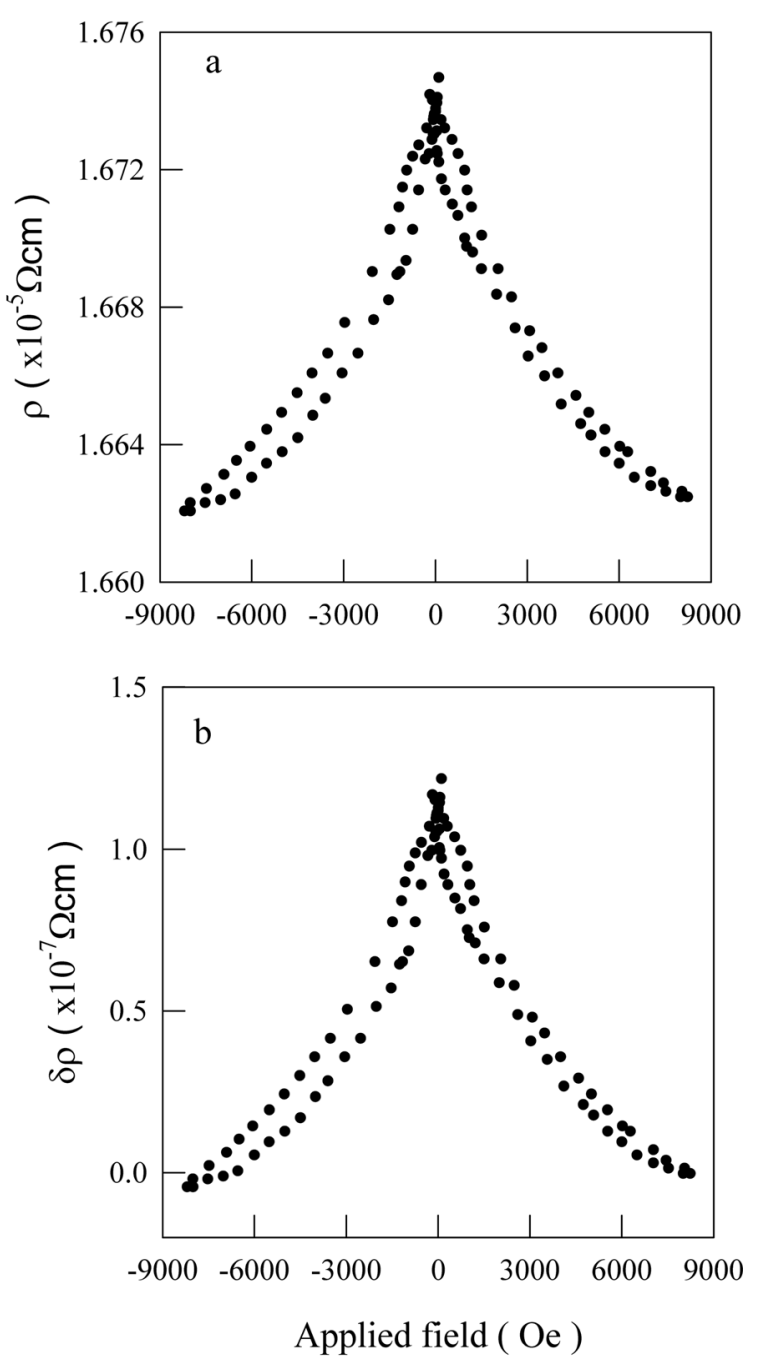

Fig. 12. The resistivity (a) and magnetoresistivity (b) changes of the $\mathrm{Co}_{0.68}-(\mathrm{TbN})_{0.32}$ film as a function of the magnetic field at room temperature.

zation was not fully saturated even with the highest applied field of $30 \mathrm{kOe}$. Figs. 12a and b show the resistivity $(\rho)$ and magnetoresistivity $(\delta \rho)$ changes of $\mathrm{Co}_{0.68}-(\mathrm{TbN})_{0.32}$ as a function of the magnetic field at room temperature. The curves show a cusp-type, negative magnetoresistance at room temperature indicating a decrease of resistivity with increasing applied field. Considering that the electrical properties of the rare-earth nitrides DyN, HoN, and ErN are all metallic [9], TbN has the same electronic structure as that of those nitrides and the $\mathrm{TbN}$ precipitate is therefore expected to also be an ordinary resistivity metal. The magnetoresistivity $(\delta \rho)$ and magnetoresistance $(\delta \rho / \rho)$ of $\mathrm{Co}_{0.68}-(\mathrm{TbN})_{0.32}$ were about $-1.12 \times 10^{-7} \Omega \mathrm{cm}$ and $0.72 \%$ at room temperature in fields up to $8 \mathrm{kOe}$, respectively (Fig. 13).

The GMR effect of the Co-TbN macroscopic ferrimag-

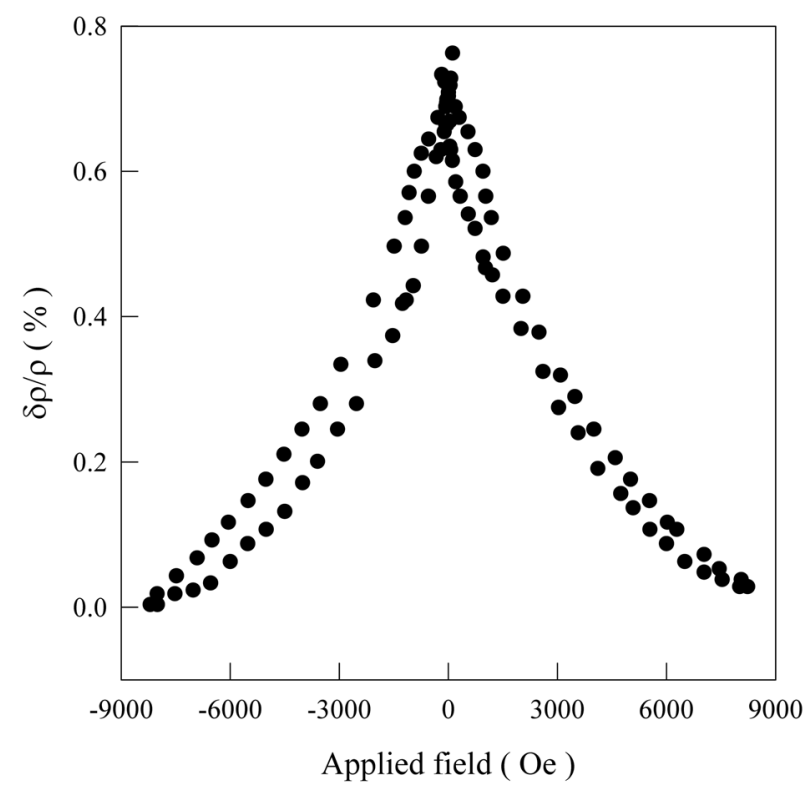

Fig. 13. The GMR $(\delta \rho / \rho)$ of the $\mathrm{Co}_{0.68}-(\mathrm{TbN})_{0.32}$ film as a function of the magnetic field at room temperature.

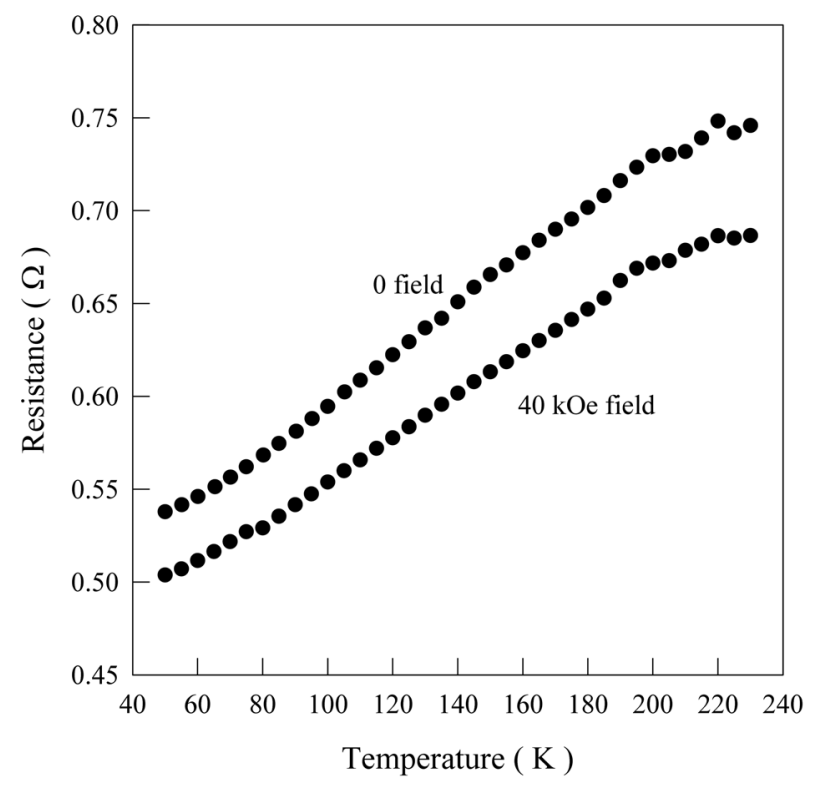

Fig. 14. The resistance changes of $\mathrm{Co}_{0.68}-(\mathrm{TbN})_{0.32}$ as a function of temperature at $\mathrm{H}=0$ and $40 \mathrm{kOe}$.

net can be described in terms of the scattering of spinpolarized, conduction electrons by the antiparallel exchange coupled spins at the phase boundary between the $\mathrm{TbN}$ precipitates and Co matrix [3]. As the matrix and precipitate in the $\mathrm{Co}-\mathrm{TbN}$ system are both metallic, the matrix is ferromagnetic and the precipitates are magnetically ordered through the exchange with the Co matrix. In the Co-EuS system, the Co matrix is metallic but the EuS in the particles is a semiconductor. Therefore, the carriers 


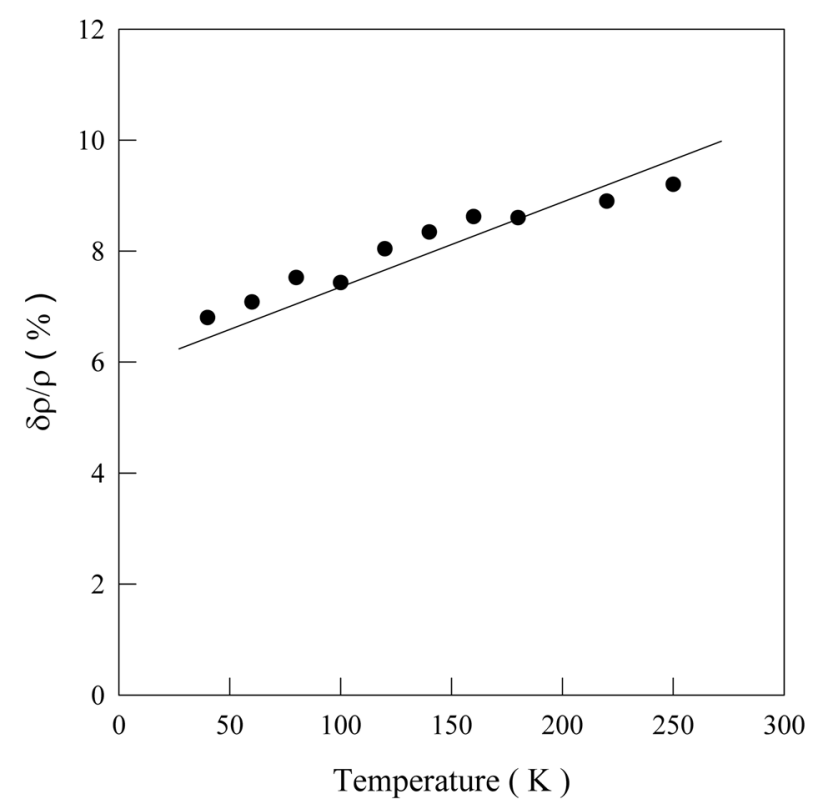

Fig. 15. The GMR of the $\mathrm{Co}_{0.68}-(\mathrm{TbN})_{0.32}$ film as a function of temperature in the high field of $40 \mathrm{kOe}$.

are mainly confined to the Co matrix, which is the main difference between the Co-EuS and Co-TbN systems. In the Co-EuS system, the conduction electrons are scattered mainly away from the Co-EuS interface, whereas in the Co-TbN system scattering can occur both at the $\mathrm{Co} / \mathrm{TbN}$ interface and in the TbN precipitates. In the Co-TbN system, when the carriers pass through the phase boundary between the two metallic phases, Co and TbN, they are scattered by the antiparallel exchange coupled spin and the resistivity is high in a zero or low field. In high fields with the $\mathrm{Co}$ and $\mathrm{TbN}$ ferromagnetically aligned, this spin scattering contribution is expected to disappear.

The resistance changes of $\mathrm{Co}_{0.68}-(\mathrm{TbN})_{0.32}$ with temperature at $\mathrm{H}=0$ and $40 \mathrm{kOe}$ displayed the typical temperature dependence of metals, as shown in Fig. 14. The GMR of $\mathrm{Co}_{0.68}-(\mathrm{TbN})_{0.32}$ in the high field of $40 \mathrm{kOe}$ was around $9 \%$ at $250 \mathrm{~K}$ (Fig. 15), due to the increased ferromagnetic alignment between the $\mathrm{Co}$ and $\mathrm{TbN}$ caused by the high field. The GMR of Co-TbN increased (Fig. 15) with increasing temperature. In contrast, ordinary GMR materials have a negative magnetoresistance which decreases with increasing temperature [10]. Based on the plot in Fig. 10 of magnetization vs. temperature in the 1 kOe magnetic field, the magnetization decreased with decreasing temperature, due to the increased magnetization of the antiparallel exchange coupled $\mathrm{TbN}$ phase. The antiparallel exchange coupling between the Co and TbN with different magnetic moments may also have strengthened with decreasing temperature. As a result, the ferrimagnetic behavior between the two different mag-

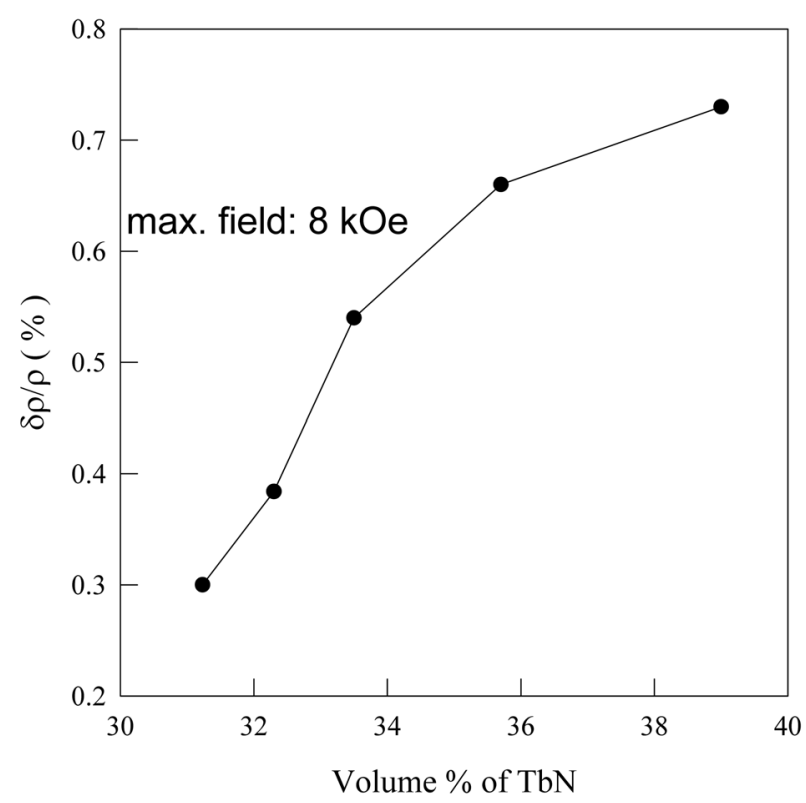

Fig. 16. The GMR changes of the Co-TbN macroscopic ferrimagnets as a function of the volume percentage of $\mathrm{TbN}$.

netic moments increased with decreasing temperature, resulting in decreased magnetization.

The variation of magnetization behavior with applied field was divided into two different regions on either side of $150 \mathrm{~K}$. By extrapolating the magnetization curves shown in Fig. 11 to higher fields, the magnetization will not be saturated in a $40 \mathrm{kOe}$ field at temperatures less than $150 \mathrm{~K}$. At high temperatures, the magnetization approached saturation in the $40 \mathrm{kOe}$ field. When the temperature increased above $150 \mathrm{~K}$, the antiparallel exchange coupling of the $\mathrm{Co}$ and $\mathrm{TbN}$ decreased with increasing temperature and the $\mathrm{Co}$ and $\mathrm{TbN}$ were more easily aligned ferromagnetically in a field. Therefore, the spin scattering contribution was expected to decrease in a high field with increasing temperature, resulting in increased magnetoresistivity $(\delta \rho)$ and $\operatorname{GMR}(\delta \rho / \rho)$ with increasing temperature.

Fig. 16 shows the dependence of GMR on the volume percentage of $\mathrm{TbN}$ calculated from the composition while assuming normal densities for $\mathrm{TbN}$ and Co. The room temperature GMR $(\delta \rho / \rho)$ of the $\mathrm{Co}_{1-\mathrm{x}}(\mathrm{TbN})_{\mathrm{x}}$ system in the $8 \mathrm{kOe}$ field increased with increasing $\mathrm{x}$. The $32 \%$ $\mathrm{TbN}$ composition with the $39 \%$ volume fraction had the largest room temperature GMR (Fig. 16).

\section{Conclusion}

A new macroscopic ferrimagnet, Co-TbN, consisting of TbN precipitates in a Co matrix, was formed by the transformation of amorphous $\mathrm{TbCo}$ into crystalline Co and 
TbN phases induced by annealing in a $\mathrm{N}_{2}$ gas atmosphere. The fully transformed films which were annealed at high temperatures demonstrated the typical macroscopic ferrimagnet properties of a magnetic compensation point and a negative GMR. The antiparallel exchange coupling at the phase boundary between the $\mathrm{TbN}$ precipitates and $\mathrm{Co}$ matrix accounted for both of these observations. The Curie temperature of $\mathrm{TbN}$ was estimated as $75 \mathrm{~K}$ from the magnetization vs. temperature curve in the $30 \mathrm{kOe}$ field. The temperature dependence of the resistivity of the macroscopic ferrimagnet $\mathrm{Co}_{0.68}-(\mathrm{TbN})_{0.32}$ exhibited the typical temperature dependence of a metal. The values of $\delta \rho$ and $\delta \rho / \rho$ in the $\mathrm{Co}_{0.68}-(\mathrm{TbN})_{0.32}$ system were maximized in the $\mathrm{TbN}$ composition range of 25 to $32 \%$. The GMR $(\delta \rho / \rho)$ of $\mathrm{Co}_{0.68}-(\mathrm{TbN})_{0.32}$ was about $9 \%$ at $250 \mathrm{~K}$ in the $40 \mathrm{kOe}$ field. The GMR effect increased with increasing temperature in the temperature range of 30 to $230 \mathrm{~K}$, due to the increased ferromagnetic alignment of the $\mathrm{Co}$ and $\mathrm{TbN}$ in the field caused by the decreased exchange coupling with increasing temperature.

\section{Acknowledgements}

This work was supported by a faculty research fund from Sejong University.

\section{References}

[1] R. J. Gambino, R. R. Ruf, and N. A. Bojarczuk, J. Appl. Phys. 75, 1871 (1994).

[2] R. J. Gambino and P. Fumagalli, IEEE Trans. Magn. 30, 4461 (1994).

[3] R. J. Gambino and J. Wang, Scr. Metall. Mater. 33, 1877 (1995).

[4] R. J. Gambino, J. Wang, and T. R. McGuire, IEEE Trans. Magn. 31, 3915 (1995).

[5] P. Fumagalli, C. Spaeth, U. Rudiger, and R. J. Gambino, IEEE Trans. Magn. 31, 3319 (1995).

[6] S. Zhang, Appl. Phys. Lett. 61, 1855 (1992).

[7] R. J. Gambino and J. J. Cuomo, J. Electrochem. Soc. 113, 401 (1966).

[8] C. Kittlel, Introduction to Solid State Physics, 7th ed., John Wiley \& Sons, Inc. (1996) p. 628.

[9] N. Sclar, J. Appl. Phys. 35, 1534 (1964).

[10] R. J. Gambino, T. R. McGuire, J. M. E. Harper, and C. Cabral, Jr., J. Appl. Phy. 75, 6909 (1994). 